\title{
Propagation Characteristics of Magneto-Ionic Plasma Columns ${ }^{1}$
}

\author{
D. Formato and A. Gilardini \\ Contribution from SELENIA S.p.A., Via Tiburtina Km 12,400, Rome, Italy
}

(Received November 29, 1961; revised March 5, 1962)

\begin{abstract}
The propagation characteristics of uniform, cylindrical plasma columns of circular cross section in axial, constant magnetic fields are determined. The ratios (plasma wavelength)/ (free-space wavelength) and (power flowing in the plasma)/(power flowing outside) are evaluated and discussed as functions of the diameter/wavelength ratio and of the plasma permittivity, for propagation of circularly symmetrical modes. Brillouin diagrams are also derived and compared with the results predicted by the quasi-static approximation. The analysis is for a nonattenuating plasma, but formulas giving the attenuation due to electronmolecule collisions are also included.
\end{abstract}

\section{Introduction}

A magneto-ionic cylindrical plasma column in a static axial magnetic field may efficiently propagate an electromagnetic wave. This fact may be of importance in interpreting phenomena like plasma oscillations and atmospheries, and in designing new methods for plasma diagnostics and millimeter wave generation.

Whereas various authors have derived the characteristic equation both for a straight column of circular cross section [Akhiezer et al., 1958] and for an infinite slab [Schumann, 1958] of uniform plasma, no detailed discussion of the propagation characteristics is given, except under the so-called "quasi-static" approximation [Smullin and Chorney, 1958; Trivelpiece and Gould, 1959].

In such an approximation the propagation wavelength must be much less than the freespace wavelength, a condition usually fulfilled only when plasma transverse dimensions are much smaller than the free-space wavelength.

Our analysis, instead, is concerned with the solution of the exact characteristic equation, written for the propagation of circularly symmetrical modes in a uniform, nonattenuating plasma of circular cross section; the permittivity tensor is chosen as constant parameter for each solution. We have found it convenient to plot our results as the ratios (plasma wavelength)/(free space wavelength) and (power flowing in the plasma)/(power flowing outside) versus the diameter/wavelength ratio.

Using these results we have also derived the plots of angular frequency versus wave number for éach mode (Brillouin diagrams), assuming constant values for the plasma and cyclotron frequencies. These diagrams are similar to those obtained using the quasi-static approximation, except for a few features, which will be described.

A final section is devoted to the attenuation formulas, when the electron-molecule collisions are taken into consideration.

\section{Plasma Dielectric Constant}

The dielectric constant of a uniform plasma in a constant magnetic field $\vec{B}$ is a tensor $\|\epsilon\|$, defined by the relationship:

$$
D_{i}=\epsilon_{i l} E_{l}
$$

${ }_{1}^{1}$ Sponsored by the Air Force Cambridge Research Center of the Air Research and Development Command, United States Air Force, through its European Office. 
where $\vec{D}$ is the electric displacement and $\vec{E}$ is the electric field.

If the field $\vec{B}$ is directed along the positive $z$ axis $(i, l=3)$, the $\epsilon_{i l}$ components due to the electron motion alone are given, for zero agitation and drift velocities, by the expressions [Allis, 1956]:

$$
\left.\begin{array}{l}
\epsilon_{11}=\epsilon_{22}=\epsilon_{0}\left(\epsilon_{1}-j \epsilon_{1}^{\prime \prime}\right)=\epsilon_{0}\left[1+\frac{\omega_{p}^{2}}{2 j \omega\left[\nu_{c}+j\left(\omega-\omega_{b}\right)\right]}+\frac{\omega_{p}^{2}}{2 j \omega\left[\nu_{c}+j\left(\omega+\omega_{b}\right)\right.}\right] \\
\epsilon_{33}=\epsilon_{0}\left(\epsilon_{3}-j \epsilon_{3}^{\prime \prime}\right)=\epsilon_{0}\left[1+\frac{\omega_{p}^{2}}{j \omega\left(\nu_{c}+j \omega\right)}\right] \\
\epsilon_{12}=-\epsilon_{21}=j \epsilon_{0}\left(\epsilon_{2}-j \epsilon_{2}^{\prime \prime}\right)=\epsilon_{0} \frac{\omega_{p}^{2}}{2 \omega}\left[\frac{1}{\nu_{c}+j\left(\omega-\omega_{b}\right)}-\frac{1}{\nu_{c}+j\left(\omega+\omega_{b}\right)}\right] \\
\epsilon_{13}=\epsilon_{23}=\epsilon_{31}=\epsilon_{32}=0 .
\end{array}\right\}
$$

Here $\nu_{c}$ is the electron collision frequency (assumed to be velocity independent), $\omega$ is the angular frequency of the applied field (well above the ion gyrofrequencies, so that the ion contributions may be neglected $), \omega_{p}$ is the angular plasma frequency $\left(=\sqrt{n e^{2} / m \epsilon_{0}}, n\right.$ being the electron density, $m$ and $e$ the electron mass and charge, and $\epsilon_{0}$ the free space permittivity) and $\omega_{b}$ is the angular cyclotron frequency $(=e B / m)$.

When $\nu_{c}^{2}<<\omega^{2}$ and $\nu_{c}^{2}<<\left(\omega-\omega_{b}\right)^{2}$, the above formulas become:

$$
\left.\begin{array}{l}
\epsilon_{1}-j \epsilon_{1}^{\prime \prime}=1+\frac{\omega_{p}^{2}}{\omega_{b}^{2}-\omega^{2}}-j \nu_{c} \frac{\omega_{p}^{2}\left(\omega_{b}^{2}+\omega^{2}\right)}{\omega\left(\omega_{b}^{2}-\omega^{2}\right)^{2}} \\
\epsilon_{2}-j \epsilon_{2}^{\prime \prime}=\frac{\omega_{b}}{\omega} \frac{\omega_{p}^{2}}{\omega_{b}^{2}-\omega^{2}}-j \nu_{c} \frac{2 \omega_{p}^{2} \omega_{b}}{\left(\omega_{b}^{2}-\omega^{2}\right)^{2}} \\
\epsilon_{3}-j \epsilon_{3}^{\prime \prime}=1-\frac{\omega_{p}^{2}}{\omega^{2}}-j \nu_{c} \frac{\omega_{p}^{2}}{\omega^{3}}
\end{array}\right\}
$$

Since the collision frequency $\nu_{c}$ is proportional to the gas pressure, the above inequalities are fulfilled, whenever the pressure is sufficiently low; we assume throughout the paper that this is always the case.

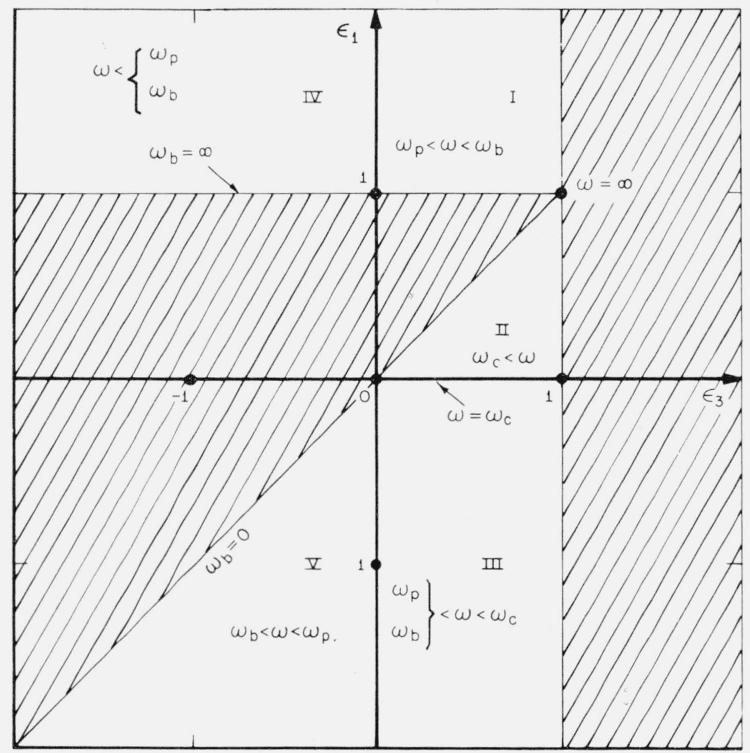

FIGURE 1. Regions of permitted and forbidden $\epsilon_{1}$ and $\epsilon_{3}$ values. 
The propagation characteristics will be first investigated assuming $\nu_{c}=0$ (and then $\epsilon_{1}^{\prime \prime}=\epsilon_{2}^{\prime \prime}=\epsilon_{3}^{\prime \prime}=0$ ), a condition which provides propagation without losses. Later we shall compute the losses, removing this assumption, but still considering the $\epsilon^{\prime \prime}$ terms small compared to the $\epsilon$ ones.

From (3) one can derive the following relationship between $\epsilon_{1}, \epsilon_{2}, \epsilon_{3}$ :

$$
\epsilon_{2}^{2}=\left(\epsilon_{1}-1\right)\left(\epsilon_{1}-\epsilon_{3}\right) \text {. }
$$

It is convenient to assume $\epsilon_{1}$ and $\epsilon_{3}$ as the independent variables of our problem. These dielectric constant components are functions of $\omega, \omega_{p}$ and $\omega_{b}$; the range of values that $\epsilon_{1}$ and $\epsilon_{3}$ may have is shown in figure 1. In this figure we have divided the plane of possible values in five regions: a separate discussion of the propagation characteristics will be presented for each region. In the figure a new convenient frequency $\omega_{c}$, defined as $\sqrt{\omega_{p}^{2}+\omega_{b}^{2}}$, has also been introduced.

\section{Propagation in a Cylindrical Column}

We assume a cylindrical plasma of circular cross section in an infinite free space. The plasma is uniform and in steady state conditions; its axis is parallel to the static magnetic field direction $(z=$ axis). Our analysis is here limited to the propagation of circularly symmetrical modes, namely to the propagation of fields without azimuthal dependence.

In a cylindrical orthogonal system of coordinates $(\rho, \varphi, z)$ these fields are [Epstein, 1956] inside the plasma:

$$
\begin{aligned}
& E_{z}=A_{1} J_{0}\left(x_{1} \rho / R\right)+A_{2} J_{0}\left(x_{2} \rho / R\right) \\
& E_{\rho}=-j \frac{2 \pi R}{\lambda_{0}}\left\{\frac{\epsilon_{2}}{\epsilon_{1}}\left[A_{1} \frac{g_{1}}{x_{1}} J_{1}\left(x_{1} \rho / R\right)+A_{2} \frac{g_{2}}{x_{2}} J_{1}\left(x_{2} \rho / R\right)\right]\right. \\
& \left.\quad-\frac{\epsilon_{3}}{\epsilon_{1}} \alpha\left[A_{1} \frac{1}{x_{1}} J_{1}\left(x_{1} \rho / R\right)+A_{2} \frac{1}{x_{2}} J_{1}\left(x_{2} \rho / R\right)\right]\right\} \\
& E_{\varphi}=\frac{2 \pi R}{\lambda_{0}}\left[A_{1} \frac{g_{1}}{x_{1}} J_{1}\left(x_{1} \rho / R\right)+A_{2} \frac{g_{2}}{x_{2}} J_{1}\left(x_{2} \rho / R\right)\right] \\
& H_{z}=j \frac{1}{Z_{0}}\left[A_{1} g_{1} J_{0}\left(x_{1} \rho / R\right)+A_{2} g_{2} J_{0}\left(x_{2} \rho / R\right)\right] \\
& H_{\rho}=-\frac{2 \pi R}{\lambda_{0}} \frac{\alpha}{Z_{0}}\left[A_{1} \frac{g_{1}}{x_{1}} J_{1}\left(x_{1} \rho / R\right)+A_{2} \frac{g_{2}}{x_{2}} J_{1}\left(x_{2} \rho / R\right)\right] \\
& H_{\varphi}=j \frac{2 \pi R}{\lambda_{0}} \frac{\epsilon_{3}}{Z_{0}}\left[A_{1} \frac{1}{x_{1}} J_{1}\left(x_{1} \rho / R\right)+A_{2} \frac{1}{x_{2}} J_{1}\left(x_{2} \rho / R\right)\right]
\end{aligned}
$$

outside the plasma:

$$
\left.\begin{array}{l}
E_{z}=B K_{0}\left(x_{0} \rho / R\right) \\
E_{\rho}=-j \frac{2 \pi R}{\lambda_{0}} \alpha \cdot B \frac{1}{x_{0}} K_{1}\left(x_{0} \rho / R\right) \\
E_{\varphi}=-\frac{2 \pi R}{\lambda_{0}} C \frac{1}{x_{0}} K_{1}\left(x_{0} \rho / R\right) \\
H_{z}=j \frac{1}{Z_{0}} C K_{0}\left(x_{0} \rho / R\right) \\
H_{\rho}=\frac{2 \pi R}{\lambda_{0}} \frac{\alpha}{Z_{0}} C \frac{1}{x_{0}} K_{1}\left(x_{0} \rho / R\right) \\
H_{\varphi}=-j \frac{2 \pi R}{x_{0}} \frac{1}{Z_{0}} B \frac{1}{x_{0}} K_{1}\left(x_{0} \rho / R\right)
\end{array}\right\}
$$


where

$R=\frac{d}{2}=$ radius of the plasma cylinder

$\alpha=\frac{\lambda_{0}}{\lambda_{g}}=$ free space $\lambda_{0}$ to guided $\lambda_{g}$ wavelength ratio

$Z_{0}=$ free space characteristic impedance

$J_{0}, J_{1}, K_{0}, K_{1}=$ Bessel functions, according to McLachlan symbolism [McLachlan, 1955].

$$
\left.\begin{array}{rl}
x_{1,2} & =\frac{2 \pi R}{\lambda_{0}} \delta_{1,2}\left(\alpha^{2}-1\right)^{\frac{1}{2}}=\delta_{1,2} x_{0} \\
x_{0} & =\frac{2 \pi R}{\lambda_{0}}\left(\alpha^{2}-1\right)^{\frac{1}{2}} \\
\delta_{1,2}^{2} & =\frac{\epsilon_{3}\left(\epsilon_{1}-\alpha^{2}\right)}{\epsilon_{1}\left(\alpha^{2}-1\right)}+\frac{\epsilon_{1}-\epsilon_{3}}{2 \epsilon_{1}}\left[-1 \pm \sqrt{1+4 \frac{\epsilon_{3}\left(\epsilon_{1}-1\right)}{\epsilon_{1}-\epsilon_{3}} \frac{\alpha^{2}}{\left(\alpha^{2}-1\right)^{2}}}\right] \\
g_{1,2} & =\frac{\epsilon_{1} \delta_{1,2}^{2}\left(\alpha^{2}-1\right)-\epsilon_{3}\left(\epsilon_{1}-\alpha^{2}\right)}{\alpha \epsilon_{2}} .
\end{array}\right\}
$$

It is desired that the fields outside the plasma decay radially to zero at infinity. Since $K_{0}$ is analogous to a negative exponential only when the argument is real positive, then $x_{0}$ must be real, and consequently $\alpha^{2}>1$.

The condition that the tangential fields be continuous at the plasma boundary gives the following transcendental equation, which determines the unknown quantity $\alpha^{2}$ :

where

$$
F\left(x_{0}, \delta_{2}, \epsilon_{3}\right)=\tau F\left(x_{0}, \delta_{1}, \epsilon_{3}\right),
$$

$$
\begin{gathered}
F\left(x_{0}, \delta, \epsilon_{3}\right)=\frac{\frac{1}{\delta} \frac{J_{1}\left(\delta x_{0}\right)}{J_{0}\left(\delta x_{0}\right)}+\frac{K_{1}\left(x_{0}\right)}{K_{0}\left(x_{0}\right)}}{\frac{\epsilon_{3}}{\delta} \frac{J_{1}\left(\delta x_{0}\right)}{J_{0}\left(\delta x_{0}\right)}+\frac{K_{1}\left(x_{0}\right)}{K_{0}\left(x_{0}\right)}} \\
\tau=\frac{g_{1}}{g_{2}}=\frac{1-\sqrt{1+4 \epsilon_{3}\left(\epsilon_{1}-1\right) \alpha^{2} /\left(\epsilon_{1}-\epsilon_{3}\right)\left(\alpha^{2}-1\right)^{2}}}{1+\sqrt{1+4 \epsilon_{3}\left(\epsilon_{1}-1\right) \alpha^{2} /\left(\epsilon_{1}-\epsilon_{3}\right)\left(\alpha^{2}-1\right)^{2}}} .
\end{gathered}
$$

Equation (8) is the characteristic equation of our propagation problem. Its solutions will be discussed in the next section.

\section{Wavelengths in Plasma Guided Propagation}

For the solution of (8) and a survey of the results it is important to know the behavior of $F\left(x_{0}, \delta, \epsilon_{3}\right)$ as a function of $x_{0}$, for different $\delta^{2}$ values. This is plotted in figure 2 and in figure 3 for positive and negative $\epsilon_{3}$ values respectively. Important points on the $x_{0}$ axis are $X_{n}^{(m)}$, $\mu^{(m)}(\delta)$, and $\nu^{(m)}\left(\delta, \epsilon_{3}\right)$, which are the $m$ th nonzero solutions of the equations:

$$
\left.\begin{array}{rl}
J_{n}\left(X_{n}^{(m)}\right) & =0 \\
\frac{1}{\delta} \frac{J_{1}\left(\mu^{(m)} \delta\right)}{J_{0}\left(\mu^{(m)} \delta\right)} & =-\frac{K_{1}\left(\mu^{(m)}\right)}{K_{0}\left(\mu^{(m)}\right)} \\
\frac{\epsilon_{3}}{\delta} \frac{J_{1}\left(\nu^{(m)} \delta\right)}{J_{0}\left(\nu^{(m)} \delta\right)} & =-\frac{K_{1}\left(\nu^{(m)}\right)}{K_{0}\left(\nu^{(m)}\right)}
\end{array}\right\}
$$


If $\delta^{2} \rightarrow+\infty$, then $\mu^{(m)}$ and $\nu^{(m)} \rightarrow X_{0}^{(m)} / \delta \rightarrow 0 . \quad$ If $\delta^{2} \rightarrow 0, \quad \mu^{(m)}$ and $\nu^{(m)}\left(\epsilon_{3}>0\right) \rightarrow X_{1}^{(m)} / \delta \rightarrow \infty$, $\nu^{(m)}\left(\epsilon_{3}<0\right) \rightarrow X_{1}^{(m-1)} / \delta \rightarrow \infty$ when $m \neq 1$, whereas $\nu^{(1)}\left(\epsilon_{3}<0\right)$ remains finite.

It is convenient to represent the solutions of (8) as relative wavelengths $\lambda_{g} / \lambda_{0}=1 / \alpha$ versus $d / \lambda_{0}$ for different sets of $\epsilon_{1}$ and $\epsilon_{3}$ values. For this purpose we have proceeded as follows:

1. From a set of real $\epsilon_{1}, \epsilon_{3}$ and $\alpha^{2}$ values the corresponding $\delta_{1}^{2}, \delta_{2}^{2}$ and $\tau$ values are computed.

2 . The $x_{0}$ solutions of (8) are determined. More than one $x_{0}$ value may be found; in this case for each solution there is a different field configuration, that is called propagation mode. Since $F\left(x_{0},+\delta, \epsilon_{3}\right)=F\left(x_{0},-\delta, \epsilon_{3}\right)$, no sign ambiguity results in taking the square root of $\delta^{2}$.

3. The corresponding $d / \lambda_{0}$ are obtained from the equation:

$$
\frac{d}{\lambda_{0}}=\frac{x_{0}}{\pi \cdot \sqrt{\alpha^{2}-1}} \cdot
$$

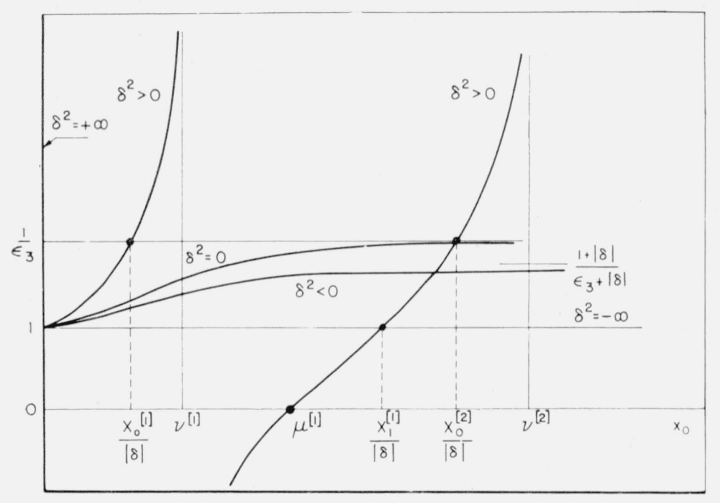

FIgURE 2. Behavior of $\mathrm{F}$ versus $\mathrm{x}_{0}$ for $\epsilon_{3}>0$ and different $\delta^{2}$ values.

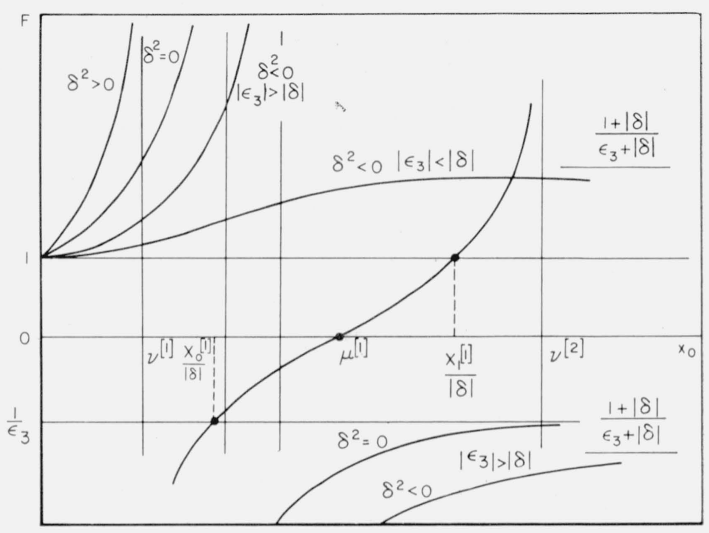

Figure 3. Behavior of $\mathrm{F}$ versus $\mathrm{x}_{0}$ for $\epsilon_{3}<0$ and different $\delta^{2}$ values.

The computations are repeated for the same $\epsilon_{1}$ and $\epsilon_{3}$, but for different $\alpha^{2}$ values between unity and infinity.

The analysis of (8) for arbitrary $\epsilon_{1}$ and $\epsilon_{3}$ values would be unnecessarily general. We have then restricted our investigation only to the sets of values, which may be found in the five significant regions shown in figure 1. The following results have been obtained.

1. In region I (see appendix $\mathrm{A}$ and fig. 4) no propagation of the $m$ th mode is possible for $d / \lambda_{0}$ values smaller than:

$$
\left(\frac{d}{\lambda_{0}}\right)_{c}^{(m)}=\frac{X_{0}^{(m)}}{\pi}\left[\frac{\left|\epsilon_{1}\right|}{\epsilon_{3}\left|\epsilon_{1}-1\right|+\sqrt{\epsilon_{3}\left(\epsilon_{1}-1\right)\left(\epsilon_{1}-\epsilon_{3}\right)}}\right]^{\frac{1}{2}}
$$

At this cutoff condition $\lambda_{g}=\lambda_{0}$; for larger $d / \lambda_{0}$ values propagation takes place with a $\lambda_{g}$ continuously decreasing towards the infinite plasma value:

$$
\lambda_{g}=\frac{\lambda_{0}}{\alpha_{0}}=\frac{\lambda_{0}}{\left[\epsilon_{1}+\sqrt{\left.\left(\epsilon_{1}-1\right)\left(\epsilon_{1}-\epsilon_{3}\right)\right]^{\frac{1}{2}}}\right.}=\frac{\lambda_{0}}{\sqrt{\epsilon_{1}+\epsilon_{2}}} .
$$

This limit represents the well-known propagation of a TEM plane uniform wave, with a righthand circular polarization. In fact, at this limit, the $E_{\rho}$ and $E_{\varphi}$ components become equal and $90^{\circ}$ out of phase. These curves have the same shape, as those for propagation in the dielectric waveguides; moreover, by setting $\epsilon_{1}=\epsilon_{3}=\epsilon$ into (13) and (14) we obtain exactly the cutoff condition and the free space propagation wavelength for dielectric waveguides, $\mathrm{TE}_{\text {om }}$ or $\mathrm{TM}_{\mathrm{om}}$ modes. 


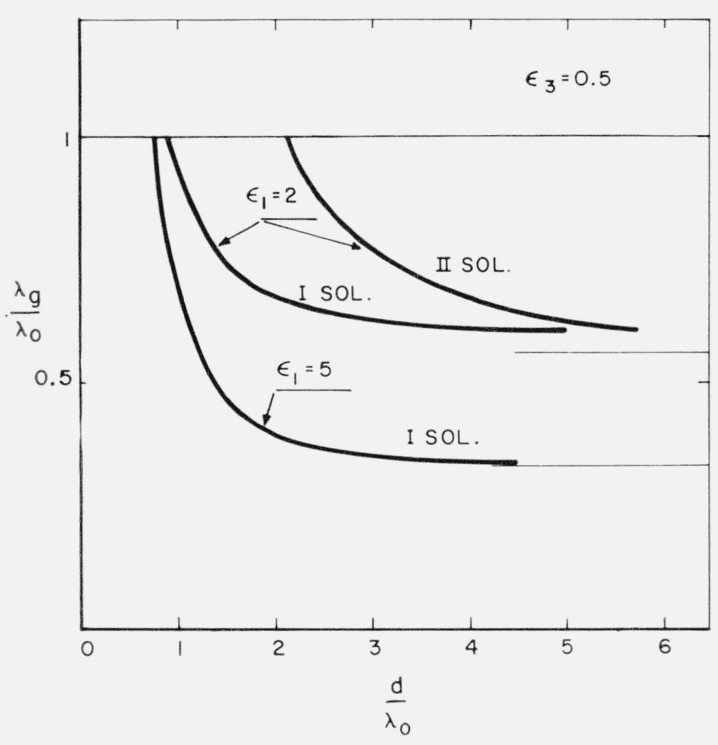

Figure 4. Wavelength ratios $\lambda_{\mathrm{g}} / \lambda_{0}$ versus $\mathrm{d} / \lambda_{0}$ in region $I$.

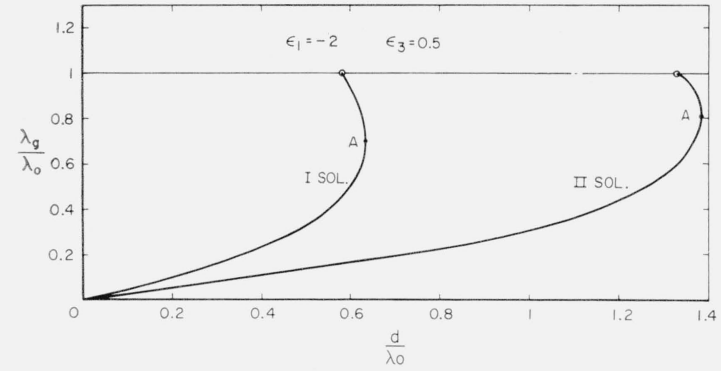

Figure 5. Wavelength ratios $\lambda_{\mathrm{g}} / \lambda_{0}$ versus $\mathrm{d} / \lambda_{0}$ in region $I I I$.

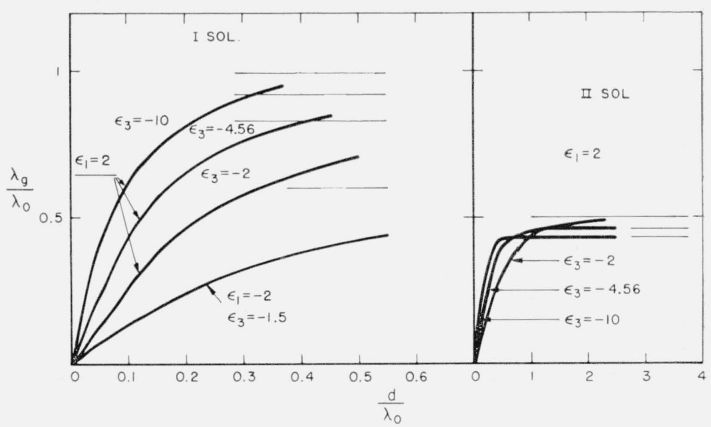

Figure 6. Wavelength ratios $\lambda_{\mathrm{g}} / \lambda_{0}$ versus $\mathrm{d} / \lambda_{0}$ in regions $I V$ and $V$.

2. In region II no propagation is ever possible (see appendix A).

3. In region III (see appendix A and fig. 5) an infinite number of propagation modes exist. All the $\lambda_{g} / \lambda_{0}$ versus $d / \lambda_{0}$ curves start from the origin, the initial slopes being $\pi / \nu^{(m)}\left(\delta_{\infty}\right)$, where $\delta_{\infty}^{2}=-\epsilon_{3} / \epsilon_{1}$. Unity wavelength ratios are attained at the same $\left(d / \lambda_{0}\right)$, defined by (13). However, when the $\lambda_{g}$ curves have the hook shape shown in figure 5, propagation takes place also at larger $d / \lambda_{0}$ ratios. In this case we have two wavelength ratios for each $d / \lambda_{0}$ condition; in general the group velocities of these two possible waves are opposite. The group velocity is zero at the maximum $d / \lambda_{0}$ (points $A$ ).

4. In region IV (see appendixes $\mathrm{B}$ and $\mathrm{C}$ and fig. 6), as in region $\mathrm{III}$, an infinite number of propagation modes exist and all the $\lambda_{g} / \lambda_{0}$ versus $d / \lambda_{0}$ curves start from the origin, the initial slopes being $\pi / \nu^{(m)}\left(\delta_{\infty}\right)$. Here, however, propagation is possible over the entire $d / \lambda_{0}$ range; the curves have positive slopes and approach, as $d / \lambda_{0} \rightarrow \infty$, an horizontal asymptote which is the same for all modes, except for the mode of the lowest $x_{0}$ solution.

5. In region $\mathrm{V}$ (see again appendixes $\mathrm{B}$ and $\mathrm{C}$ and fig. 6 ) we have at most one $\lambda_{g} / \lambda_{0}$ versus $d / \lambda_{0}$ curve for each pair of $\epsilon_{1}, \epsilon_{3}$ values. The curve has the same behavior as the curves found in region IV. The initial slope is $\pi / \nu^{(1)}\left(\delta_{\infty}\right)$. The curve may exist only if $\epsilon_{1} \epsilon_{3}>1$, which in the frequency domain corresponds to the $\omega_{b}<\omega<\omega_{c} / \sqrt{2}<\omega_{p}$ range.

The above discussed results coincide, in the $\lambda_{g} / \lambda_{0}<<1$ region, with those given by the quasistatic approximation. 


\section{Brillouin Diagrams}

Keeping $\epsilon_{1}$ and $\epsilon_{3}$ as constant parameters in the Maxwell equations, it has been possible to solve the dispersion equation using a lengthy but straightforward procedure. Brillouin diagrams $\left(\beta=\frac{2 \pi}{\lambda_{g}}\right.$ versus $k=2 \pi / \lambda_{0}=\omega / c, c$ being the light velocity) are, instead, more difficult to derive, because $\epsilon_{1}$ and $\epsilon_{3}$ are functions of frequency.

In order to obtain these diagrams from the previous section curves we must solve the following system:

$$
\left.\begin{array}{rl}
\lambda_{g} / \lambda_{0} & =f\left(d / \lambda_{0}, \epsilon_{1}, \epsilon_{3}\right) \\
\epsilon_{1} & =\left(1+\frac{\omega_{p}^{2}}{\omega_{b}^{2}-\omega^{2}}\right) \\
\epsilon_{3} & =\left(1-\frac{\omega_{p}^{2}}{\omega^{2}}\right) .
\end{array}\right\}
$$

For this let us keep constant the ratio $a=\frac{\omega_{b}^{2}}{\omega_{p}^{2}}$ and choose all the pairs of $\epsilon_{1}$ and $\epsilon_{3}$ values, which satisfy the relationship:

$$
\epsilon_{1}=\frac{a\left(1-\epsilon_{3}\right)-\epsilon_{3}}{a\left(1-\epsilon_{3}\right)-1}
$$

Then the first equation of the system (15) provides, in the Brillouin $k d$ versus $\beta d$ plane, a set of curves, $\epsilon_{3}$, and $a$ being constant for each curve. These curves will intercept the horizontal lines:

$$
k d=k_{p} d / \sqrt{1-\epsilon_{3}}
$$

which represent the third equation of the above system (15), $k_{p} d$ being the plasma density parameter $\omega_{p} d / c$. For given values of the frequency independent parameters $a$ and $k_{p} d$, these intercepts at different $\epsilon_{3}$ values describe the Brillouin curves:

$$
\beta d=F\left(k d, a, k_{p} d\right) .
$$

Typical Brillouin diagrams are plotted in figures 7 and 8 for $k_{p} d=3.5$ and for two $a$ values, the first chosen in the $a>1$ region $(a=2.25)$, the second in the $a<1$ region $(a=0.5)$.

It is interesting to compare these curves with the corresponding curves obtained by Gould and Trivelpiece [1959] and by Smullin and Chorney [1958] using the so-called quasistatic approximation. These authors have studied the more general case of a circular waveguide partially filled with plasma; from their results the curves to be used in our case are easily derived by setting the waveguide diameter equal to infinity.

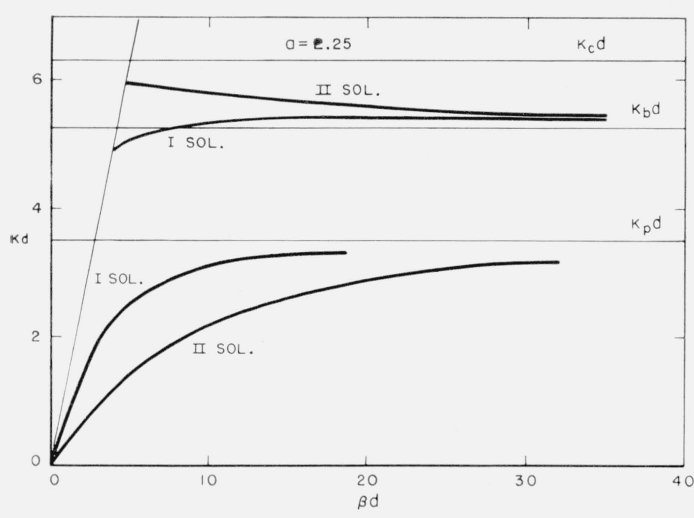

Figure 7. Brillouin diagrams for $\mathrm{a}=2.25$ and $\mathrm{k}_{\mathrm{p}} \mathrm{d}=$ 3.5 .

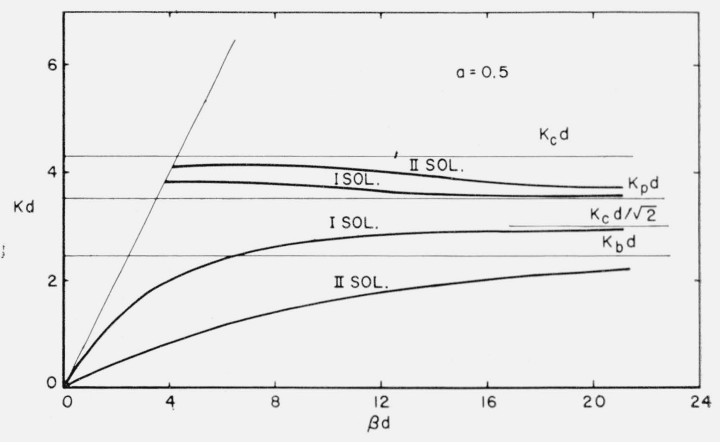

Figure 8. Brillouin diagrams for $\mathrm{a}=0.5$ and $\mathrm{k}_{\mathrm{p}} \mathrm{d}=3.5$. 
No significant difference is found in the behavior of the Brillouin diagrams in the $\omega<\omega_{p}$ region $\left(k d<k_{p} d\right)$. In this region all the $k d$ versus $\beta d$ curves start from the origin and approach asymptotically the value $k_{p} d$ or $k_{b} d$, whichever is smaller, except the first solution mode which, when $\omega_{p}>\omega_{b}$, approaches the asymptotic value $k_{c} d / \sqrt{2}=\left(k_{p}^{2} d^{2}+k_{b}^{2} d^{2}\right)^{\frac{1}{2}} / \sqrt{2}$.

A behavior slightly more complicated than predicted from the quasi-static approximation is found in the $\omega>\omega_{p}$ region. Here the curves start from different points of the $k d=\beta d$ line between $k_{p} d$ and $k_{c} d$ and approach the asymptotic value $k_{p} d$ or $k_{b} d$, whichever is larger. The slopes of these curves may be positive, negative, or partially positive and partially negative, so that the waves can be forward or backward. It is worth noting that in the quasi-static approximation all these waves are always backward.

The following details can be added to the discussed features in the $\omega>\omega_{p}$ region.

(a) The starting points on the $k d=\beta d$ line are given from the simple relation:

where

$$
k d=k_{p} d \frac{\sqrt{a+(1+p)^{2}}}{1+p}
$$

$$
p=\left[k_{p} d / 2 X_{0}^{(m)}\right]^{2} .
$$

(b) When $\omega_{b}>\omega_{p}$ the wave of a given mode is always forward if:

$$
k_{b}^{2} d^{2}-k_{p}^{2} d^{2}>2\left(X_{1}^{(m)}\right)^{2} .
$$

If this condition is not satisfied, the wave may be backward, or start at low $\beta d$ values as forward and change to backward at large $\beta d$ values.

For a plasma, which, instead of being stationary, has a uniform drift motion $u_{0}$ along the $z$ axis, the Brillouin diagrams are obtained from the above ones by substituting the Doppler shifted frequency $\omega-\beta u_{0}$ to the signal frequency $\omega$. Our results are thus translated into the phase characteristics of space charge and transverse field waves of an electron beam. In this way they are useful for studies of traveling-wave and parametric amplification tubes.

\section{Power Flow}

The power flowing inside the plasma and the power flowing outside are obtained by integration of the Poynting vector over the respective areas.

The $z$ component of this vector inside the plasma is

$$
S_{z}^{p}=\frac{\alpha D^{2}}{Z_{0}}\left(\frac{2 \pi R}{\lambda_{0}}\right)^{2}\left[\frac{N_{1} G_{1}^{2}}{g_{1}^{2} x_{1}^{2}} \frac{J_{1}^{2}\left(x_{1} \rho / R\right)}{J_{0}^{2}\left(x_{1}\right)}+\frac{N_{2} G_{2}^{2}}{g_{2}^{2} x_{2}^{2}} \frac{J_{1}^{2}\left(x_{2} \rho / R\right)}{J_{0}^{2}\left(x_{2}\right)}-\frac{N_{3} G_{1} G_{2}}{g_{1} g_{2} x_{1} x_{2}} \frac{J_{1}\left(x_{1} \rho / R\right)}{J_{0}\left(x_{1}\right)} \frac{J_{1}\left(x_{2} \rho / R\right)}{J_{0}\left(x_{2}\right)}\right]
$$

where, for symmetry and simplicity, we have introduced the new symbols:

$$
\left.\begin{array}{rl}
D & =\frac{g_{1}}{G_{1}} J_{0}\left(x_{1}\right) A_{1} \\
G_{1} & =\left[\frac{1}{x_{1}} \frac{J_{1}\left(x_{1}\right)}{J_{0}\left(x_{1}\right)}+\frac{1}{x_{0}} \frac{K_{1}\left(x_{0}\right)}{K_{0}\left(x_{0}\right)}\right]^{-1} \\
G_{2} & =\left[\frac{1}{x_{2}} \frac{J_{1}\left(x_{2}\right)}{J_{0}\left(x_{2}\right)}+\frac{1}{x_{0}} \frac{K_{1}\left(x_{0}\right)}{K_{0}\left(x_{0}\right)}\right]^{-1} \\
N_{1,2} & =g_{1,2}^{2}+\frac{\epsilon_{3}^{2}}{\epsilon_{1}}-\frac{\epsilon_{3} \epsilon_{2}}{\epsilon_{1}} \frac{g_{1,2}}{\alpha}=g_{1,2}^{2}-\frac{\epsilon_{3}\left(\alpha^{2}-1\right) \delta_{1,2}^{2}-\epsilon_{3}^{2}}{\alpha^{2}} \\
N_{3} & =2 g_{1} g_{2}+2 \frac{\epsilon_{3}^{2}}{\epsilon_{1}}-\frac{\epsilon_{3} \epsilon_{2}}{\epsilon_{1}} \frac{g_{1}+g_{2}}{\alpha}=-\frac{\epsilon_{3}\left(\epsilon_{1}-\epsilon_{3}\right)}{\epsilon_{1}} \frac{\alpha^{2}+1}{\alpha^{2}} .
\end{array}\right\}
$$

The $z$ component of the Poynting vector outside the plasma is

$$
S_{z}^{a}=\frac{\alpha D^{2}}{Z_{0}}\left(\frac{2 \pi R}{\lambda_{0}}\right)^{2}\left[\left(\frac{G_{1}}{g_{1}}-\frac{G_{2}}{g_{2}}\right)^{2}+\left(G_{1}-G_{2}\right)^{2}\right] \cdot \frac{1}{x_{0}^{2}} \frac{K_{1}^{2}\left(x_{0} \rho / R\right)}{K_{0}^{2}\left(x_{0}\right)} .
$$


When integrations are performed, the ratio between the inside and outside powers can be written as:

$$
\frac{P_{p}}{P_{a}}=\frac{\int_{0}^{R} S_{z}^{p} \rho d \rho}{\int_{R}^{\infty} S_{z}^{a} \rho d \rho}=\left(\frac{P_{p}}{P_{a}}\right)_{1}+\left(\frac{P_{p}}{P_{a}}\right)_{2}+\left(\frac{P_{p}}{P_{a}}\right)_{3}
$$

where

$$
\left.\begin{array}{rl}
\left(\frac{P_{p}}{P_{a}}\right)_{1} & =\frac{N_{1} G_{1}^{2}}{\delta_{1}^{2} g_{1}^{2} M}\left(1-\frac{2}{x_{1}} \frac{J_{1}\left(x_{1}\right)}{J_{0}\left(x_{1}\right)}+\frac{J_{1}^{2}\left(x_{1}\right)}{J_{0}^{2}\left(x_{1}\right)}\right) \\
\left(\frac{P_{p}}{P_{a}}\right)_{2} & =\frac{N_{2} G_{2}^{2}}{\delta_{2}^{2} g_{2}^{2} M}\left(1-\frac{2}{x_{2}} \frac{J_{1}\left(x_{2}\right)}{J_{0}\left(x_{2}\right)}+\frac{J_{1}^{2}\left(x_{2}\right)}{J_{0}^{2}\left(x_{2}\right)}\right) \\
\left(\frac{P_{p}}{P_{a}}\right)_{3} & =\frac{2 N_{3}\left(G_{1}-G_{2}\right)}{\left(\delta_{1}^{2}-\delta_{2}^{2}\right) g_{1} g_{2} M} \\
M & =\left[\left(\frac{G_{1}}{g_{1}}-\frac{G_{2}}{g_{2}}\right)^{2}+\left(G_{1}-G_{2}\right)^{2}\right]\left(1+\frac{2}{x_{0}} \frac{K_{1}\left(x_{0}\right)}{K_{0}\left(x_{0}\right)}-\frac{K_{1}^{2}\left(x_{0}\right)}{K_{0}^{2}\left(x_{0}\right)}\right)
\end{array}\right\}
$$

The three terms of (24) are respectively proportional to the power transported by the field components of amplitude $A_{1}$, by the field components of amplitude $A_{2}$ and by their mutual interaction (with reference to the expressions (5)).

The $P_{p} / P_{a}$ ratio is always real, also in the region where $\delta_{1}^{2}$ and $\delta_{2}^{2}$ are complex; in fact, in this region $x_{1}$ and $x_{2}, g_{1}$ and $g_{2}, N_{1}$ and $N_{2}, G_{1}$ and $G_{2}$ are all complex conjugates, so that the resulting $\left(P_{p} / P_{a}\right)_{1}$ and $\left(P_{p} / P_{a}\right)_{2}$ are complex conjugates too, and their sum is real, as it is the third term $\left(P_{p} / P_{a}\right)_{3}$.

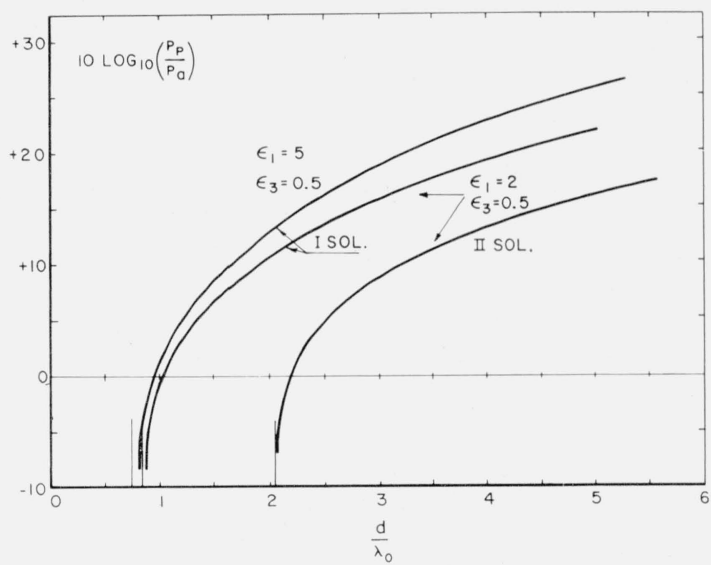

Figure 9. Power flow ratios $\mathrm{P}_{\mathrm{p}} / \mathrm{P}_{\mathrm{a}}$ versus $\mathrm{d} / \lambda_{0}$ in region $I$.

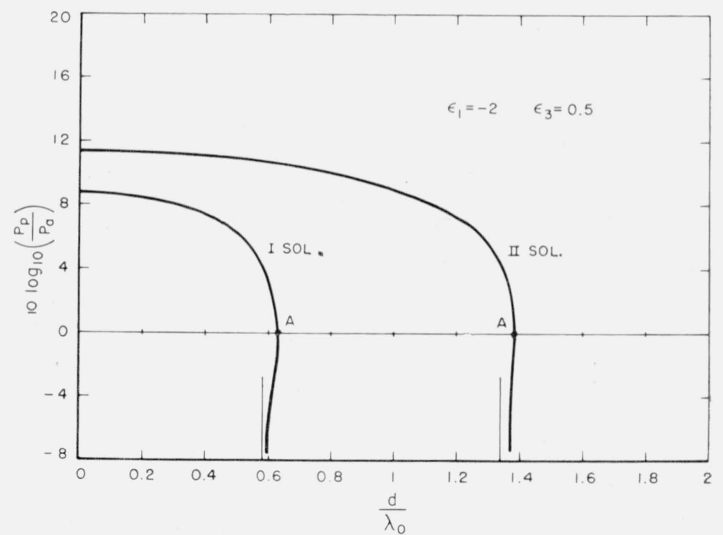

Figure 10. Power flow ratios $\mathrm{P}_{\mathrm{p}} / \mathrm{P}_{\mathrm{a}}$ versus $\mathrm{d} / \lambda_{0}$ in region $I I I$.

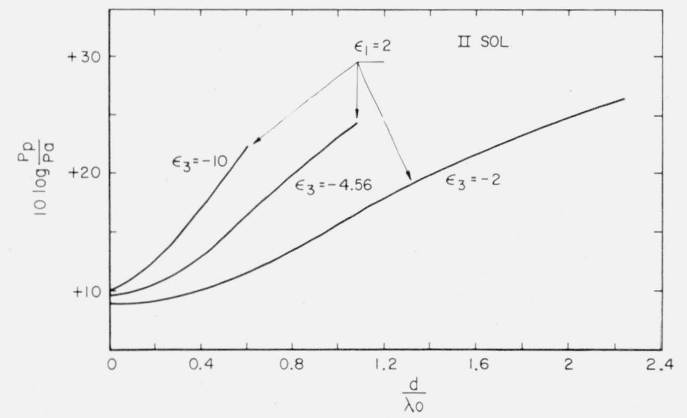

Figure 11. Power flow ratios $\mathrm{P}_{\mathrm{p}} / \mathrm{P}_{\mathrm{a}}$ versus $\mathrm{d} / \lambda_{0}$ in region $I V$. 
The ratios $P_{p} / P_{a}$ versus $d / \lambda_{0}$ have been computed and plotted in figures 9,10 , and 11 for the same $\epsilon_{1}$ and $\epsilon_{3}$ values used in the numerical solutions of the characteristic equation, with the exception of the first solution in the $\epsilon_{3}<0$ region.

The ratios for this first solution have been evaluated in the limiting cases of zero and infinite $d / \lambda_{0}$ values and have resulted much smaller than unity. The practical interest of this mode seems then very scarce; for this reason the complete curves have not been evaluated.

A few more remarks seem worth mentioning:

1. The Poynting vector has also a nonzero $\phi$ component, so that the power is actually flowing helically around the $z$ direction.

2. In region III the power flow inside the plasma may be backward. In this case, if $P_{p}>P_{a}$ we have an "average" backward wave, if $P_{p}<P_{a}$ an "average" forward wave. The points labeled $\mathrm{A}$ in figure 5, where the group velocity is zero, correspond to the points $\mathrm{A}$ in figure 10 where $P_{p}=P_{a}$, so that, although power is actually flowing separately inside and outside the plasma, the total flow is zero.

3. Also in region $\mathrm{V}$ the power flow in the plasma may be backward, although the total power is flowing forward, being $P_{p} P_{a}$.

\section{Attenuation Coefficient}

The attenuation coefficient $a$ for the wave propagation is related to the power dissipated by losses per unit length $P_{d}$ by the well-known formula:

$$
a=\frac{1}{2} \frac{P_{d}}{P_{p}+P_{a}} .
$$

We neglect the losses in the dielectric surrounding the plasma (usually air) and assume that the fields are those we have computed in the previous paragraphs, neglecting losses. In this case the losses are only due to the imaginary terms of the plasma dielectric constant and $P_{d}$ is given by:

$$
P_{d}=\operatorname{Real}\left[j \omega \int_{0}^{R} \vec{E} \cdot \vec{D}^{*} 2 \pi \rho d \rho\right]
$$

By means of (1) and (2), and recalling that $E_{\rho}$ and $E_{\varphi}$ are $90^{\circ}$ out of phase, this becomes:

$$
P_{d}=\omega \epsilon_{0} \int_{0}^{R}\left[\epsilon_{1}^{\prime \prime}\left(E_{\rho} E_{\rho}^{*}+E_{\varphi} E_{\varphi}^{*}\right)-j \epsilon_{2}^{\prime \prime}\left(E_{\rho} E_{\varphi}^{*}-E_{\varphi} E_{\rho}^{*}\right)+\epsilon_{3}^{\prime \prime} E_{z} E_{z}^{*}\right] 2 \pi \rho d \rho
$$

Substituting the field expressions and performing the integrations, we finally obtain:

$$
a=\frac{\pi}{\lambda_{0}}\left\{\frac{W_{1}}{N_{1}}\left(\frac{P_{p}}{P_{a}}\right)_{1}+\frac{W_{2}}{N_{2}}\left(\frac{P_{p}}{P_{a}}\right)_{2}+\frac{W_{3}}{N_{3}}\left(\frac{P_{p}}{P_{a}}\right)_{3}+\epsilon_{3}^{\prime \prime} \frac{Y}{M} \frac{\alpha^{2}-1}{\alpha}\right\}\left(1+\frac{P_{p}}{P_{a}}\right)^{-1}
$$

where:

$$
\left.\begin{array}{rl}
W_{1} & =\epsilon_{1}^{\prime \prime}\left[\left(\frac{\epsilon_{2}^{2}}{\epsilon_{1}^{2}}+1\right) \frac{g_{2}^{2}}{\alpha}+\frac{\epsilon_{3}^{2}}{\epsilon_{1}^{2}} \alpha-2 \frac{\epsilon_{2} \epsilon_{3}}{\epsilon_{1}^{2}} g_{1}\right]-2 \epsilon_{2}^{\prime \prime}\left[\frac{\epsilon_{2}}{\epsilon_{1}} \frac{g_{2}^{2}}{\alpha}-\frac{\epsilon_{3}}{\epsilon_{1}} g_{1}\right] \\
W_{2} & =\epsilon_{1}^{\prime \prime}\left[\left(\frac{\epsilon_{2}^{2}}{\epsilon_{1}^{2}}+1\right) \frac{g_{2}^{2}}{\alpha}+\frac{\epsilon_{3}^{2}}{\epsilon_{1}^{2}} \alpha-2 \frac{\epsilon_{2} \epsilon_{3}}{\epsilon_{1}^{2}} g_{2}\right]-2 \epsilon_{2}^{\prime \prime}\left[\frac{\epsilon_{2}}{\epsilon_{1}} \frac{g_{1}^{2}}{\alpha}-\frac{\epsilon_{3}}{\epsilon_{1}} g_{2}\right] \\
W_{3} & =2 \epsilon_{1}^{\prime \prime}\left[\left(\frac{\epsilon_{2}^{2}}{\epsilon_{1}^{2}}+1\right) \frac{g_{1} g_{2}}{\alpha}+\frac{\epsilon_{3}^{2}}{\epsilon_{1}^{2}} \alpha-\frac{\epsilon_{2} \epsilon_{3}}{\epsilon_{1}^{2}}\left(g_{1}+g_{2}\right)\right]-4 \epsilon_{2}^{\prime \prime}\left[\frac{\epsilon_{2}}{\epsilon_{1}} \frac{g_{1} g_{2}}{\alpha}-\frac{\epsilon_{3}}{2 \epsilon_{1}}\left(g_{1}+g_{2}\right)\right] \\
Y & =\frac{G_{1}^{2}}{g_{1}^{2}}\left[1+\frac{J_{1}^{2}\left(x_{1}\right)}{J_{0}^{2}\left(x_{1}\right)}\right]+\frac{G_{2}^{2}}{g_{2}^{2}}\left[1+\frac{J_{1}^{2}\left(x_{2}\right)}{J_{0}^{2}\left(x_{2}\right)}\right]-\frac{4 G_{1} G_{2}}{g_{1} g_{2}} \frac{x_{1}}{\frac{J_{1}\left(x_{1}\right)}{J_{0}\left(x_{1}\right)}-x_{2} \frac{J_{1}\left(x_{2}\right)}{J_{0}\left(x_{2}\right)}} . x_{1}^{2}-x_{2}^{2}
\end{array}\right\}
$$




\section{Conclusion}

Beside giving procedure for the solution of the characteristic equation of a uniform magneto-ionic plasma column of circular cross section and computing the $\lambda_{g} / \lambda_{0}$ and $P_{p} / P_{a}$ values for a few typical cases, our analysis has provided the following results, not given by the previous quasi-static analyses:

(1) Propagation may take place in the $\omega_{p}<\omega<\omega_{b}$ region, provided $d / \lambda_{0}$ is larger than a minimum cutoff value; in this case, at $d / \lambda_{0}$ values sufficiently larger than the cutoff value, power flow takes place mainly in the plasma $\left(P_{p} / P_{a}>1\right)$.

2. In the $\omega>\omega_{b}, \omega_{p}$ region we may have not only backward waves, but also forward waves. Backward waves carry most of the power inside the plasma. Here too propagation is possible only over a finite range of $d / \lambda_{0}$ values.

3. When $\omega<\omega_{p}\left(\epsilon_{3}<0\right)$, in the ranges where computations have been performed, power flow in the first circularly symmetrical mode takes place mainly outside the plasma, whereas all other circularly symmetrical modes show large $P_{p} / P_{a}$ ratios.

The authors thank A. Lemma, who carried out the numerical computations, applying also electronic computing techniques. The valuable help of Dr. A. Boschi is also acknowledged.

\section{Appendix A}

Regions I, II, and III of figure 1 have in common the following features:

(a) $0<\epsilon_{3}<1$;

(b) $\delta_{1}^{2}$ and $\delta_{2}^{2}$ are always real;

(c) $\delta_{2}^{2} \leq-1\left(\delta_{2}^{2}\right.$ versus $\alpha$ rises steadily from $-\infty$ at $\alpha=1$ to -1 as $\left.\alpha \rightarrow \infty\right)$;

(d) $-1 \leq \tau \leq 0$ ( $\tau$ versus $\alpha$ rises steadily from -1 at $\alpha=1$ to 0 as $\alpha \rightarrow \infty$ ).

Inspection of figure 2 shows that in this case:

(a) When $\delta_{1}^{2}>0$ there are an infinite number of $x_{0}$ solutions of (8); the $m$ th solution falls between $\nu^{(m)}\left(\delta_{1}\right)$ and $\mu^{(m)}\left(\delta_{1}\right)$. As $\delta_{1}^{2} \rightarrow+\infty$ this solution vanishes $\left(x_{0} \sim X_{0}^{(m)} / \delta_{1}\right)$ and as $\delta_{1}^{2} \rightarrow 0$ it becomes infinite $\left(x_{0} \simeq X_{1}^{(m)} / \delta_{1}\right)$. In the $\tau=0$ limit $(\alpha \rightarrow \infty)$ the $x_{0}$ solutions become equal to $\nu^{(m)}\left(\delta_{1}\right)$.

(b) When $\delta_{1}^{2}<0$, no solutions of (8) exist.

The above results determine the behavior of the $x_{0}$ versus $\alpha$ solutions, for given $\epsilon_{1}$ and $\epsilon_{3}$, if we know the characteristics of the $\delta_{1}^{2}$ versus $\alpha$ curves. These are as follows:

(a) In region $I, \delta^{2}$ drops from $+\infty$ at $\alpha=1$ to 0 at $\alpha_{0}$ :

$$
\alpha_{0}^{2}=\epsilon_{1}+\sqrt{\left(\epsilon_{1}-1\right)\left(\epsilon_{1}-\epsilon_{3}\right)}=\epsilon_{1}+\epsilon_{2} .
$$

The parameter $\delta_{1}^{2}$ becomes negative, when $\alpha>\alpha_{0}$.

(b) In region $\mathrm{II}, \delta_{1}^{2}$ is always negative.

(c) In region III, $\delta_{1}^{2}$ is always positive and drops from $+\infty$ at $\alpha=1$ to $\delta_{\infty}^{2}=-\frac{\epsilon_{3}}{\epsilon_{1}}>0$ as $\alpha \rightarrow \infty$.

\section{Appendix B}

Regions IV and V, when:

$$
\alpha^{2} \geq \alpha_{i}^{2}=\left(\epsilon_{1}-\epsilon_{3}\right)^{-1}\left(\epsilon_{1}+\epsilon_{3}-2 \epsilon_{1} \epsilon_{3}+2 \sqrt{\epsilon_{1} \epsilon_{3}\left(\epsilon_{1}-1\right)\left(\epsilon_{3}-1\right)}\right)
$$

have in common the following features:

(a) $\epsilon_{3}<0$;

(b) $\delta_{1}^{2}$ and $\delta_{2}^{2}$ are always real;

(c) $\delta_{1}^{2}$ and $\delta_{2}^{2}$ versus $\alpha$ are respectively the upper and lower branches of a curve, the shape of which is that of a deformed parabola with the vertex at $\alpha_{i}$ and two horizontal asymptots for $\alpha \rightarrow \infty\left(\delta_{1}^{2} \rightarrow \delta_{\infty}^{2}=-\epsilon_{3} / \epsilon_{1}\right.$ and $\left.\delta_{2}^{2} \rightarrow-1\right)$.

(d) $0 \leq \tau \leq 1$ ( $\tau$ versus $\alpha$ drops from 1 at $\alpha_{i}$ to 0 as $\left.\alpha \rightarrow \infty\right)$. 
Inspection of figure 3 shows that in this case:

(a) When $\delta_{1}^{2}>0$, there is an infinite number of solutions. The smallest $x_{0}$ (first solution) falls between zero and $\nu^{(1)}\left(\delta_{1}\right)$; as $\delta_{1} \rightarrow 0$, it remains then finite. All the other $x_{0}$ solutions rise instead to infinity as $\delta_{1}^{2} \rightarrow 0$, because the $m$ th solution always falls between $X_{1}^{(m-1)} / \delta_{1}$ and $X_{0}^{(m)} / \delta_{1}$, if $\delta_{2}^{2}<0$. In the $\tau=0$ limit $(\alpha \rightarrow \infty)$ the $x_{0}$ solutions become equal to $\nu^{(m)}\left(\delta_{\infty}\right)$. In the $\tau=1$ limit $\left(\alpha=\alpha_{i}, \delta_{1}^{2}=\delta_{2}^{2}\right.$ ), all the solutions go to infinity, except the first one which remains finite; in this limit (8) becomes an identity and the solution is given by the equation:

$$
[\partial(g F) / \partial \delta]_{\alpha=\alpha}=0 .
$$

(b) When $-\epsilon_{3}^{2} \leq \delta_{1}^{2}<0$, there is always one solution alone. This solution falls between zero and $\nu^{(1)}\left(\delta_{1}\right)$, and becomes equal to $\nu^{(1)}\left(\delta_{\infty}\right)$, as $\tau \rightarrow 0$.

(c) When $\delta_{1}^{2}<-\epsilon_{3}^{2}$, there is one solution or no solutions depending on $\tau$ being larger or smaller than:

$$
\tau_{0}=\frac{1+\left|\delta_{2}\right|}{1+\left|\delta_{1}\right|} \frac{\epsilon_{3}+\left|\delta_{1}\right|}{\epsilon_{3}+\left|\delta_{2}\right|}
$$

As $\tau \rightarrow \tau_{0}, x_{0} \rightarrow \infty$.

Here too the $x_{0}$ versus $\alpha$ behavior can be derived from the above results, whenever the characteristics of the $\delta_{1}^{2}$ versus $\alpha$ curves are known. The basic features of these curves are

(a) In region IV $\delta_{\infty}^{2}>0$, so that there is always one region extending up to $\alpha=\infty$, where $\delta_{1}^{2}>0$. This region is the entire $\alpha \geq \alpha_{i}$ range, when:

$$
\epsilon_{3} \leq \epsilon_{3}^{0}=\frac{1}{2}\left(1-3 \epsilon_{1}-\sqrt{9 \epsilon_{1}^{2}-10 \epsilon_{1}+1}\right)
$$

and is the $\alpha \geq \alpha_{0}$ range [see $(\mathrm{A}-1)$ ], when $\epsilon_{3}>\epsilon_{3}^{0}$.

(b) In region $\mathrm{V}, \delta_{\infty}^{2}<0$ and then $\delta_{1}^{2}<0$ always. When $\delta_{\infty}^{2}<-\epsilon_{3}^{2}$, no solutions exist as $\alpha \rightarrow \infty$, because $\tau=0<\tau_{0}$; in this case, continuity and regularity suggest, as confirmed by extensive numerical computations, that no solutions exist at any $\alpha$ value. Propagation is then possible only if $\epsilon_{1} \epsilon_{3}>1$ namely in the region:

$$
\left\{\begin{array}{l}
-\infty<\epsilon_{1}<-1 \\
\epsilon_{1}<\epsilon_{3}<1 / \epsilon_{1}
\end{array}\right.
$$

\section{Appendix C}

Regions IV and V, when $\alpha^{2}<\alpha_{i}^{2}$, have in common the following features:

(a) $\epsilon_{3}<0$;

(b) $\delta_{1}^{2}$ and $\delta_{2}^{2}$ are complex conjugates;

(c) $\tau$ is a complex number, and $|\tau|=1$.

It is convenient to transform (8) into an equation of only real quantities. For this purpose, recalling that Bessel functions of complex conjugate arguments are complex conjugates, we write:

$$
\left.\begin{array}{l}
\delta_{1,2}^{2}=|\delta|^{2} \exp ( \pm 2 j \theta) \\
g_{1,2}=|g| \exp ( \pm j \vartheta) \\
J_{0}\left(\delta_{1,2} x_{0}\right)=S_{0}\left(|\delta| x_{0}, \theta\right) \exp \left[ \pm j \psi_{0}\left(|\delta| x_{0}, \theta\right)\right] \\
J_{1}\left(\delta_{1,2} x_{0}\right)=S_{1}\left(|\delta| x_{0}, \theta\right) \exp \left[ \pm j \psi_{1}\left(|\delta| x_{0}, \theta\right)\right] .
\end{array}\right\}
$$


Equation (8) becomes:

$$
\frac{\epsilon_{3}}{|\delta|^{2}} \frac{S_{1}^{2}}{S_{0}^{2}}+\frac{K_{1}^{2}\left(x_{0}\right)}{K_{0}^{2}\left(x_{0}\right)}=-\frac{1}{|\delta|} \frac{S_{1}}{S_{0}} \frac{K_{1}\left(x_{0}\right)}{K_{0}\left(x_{0}\right)}\left[\frac{\epsilon_{3} \sin \left[(\vartheta+\Theta)-\left(\psi_{1}-\psi_{0}\right)\right]}{\sin \vartheta}+\frac{\sin \left[(\vartheta-\Theta)+\left(\psi_{1}-\psi_{0}\right)\right]}{\sin \vartheta}\right] .
$$

When an $x_{0}$ solution exists in the $\alpha>\alpha_{i}$ region and it ends at $\alpha_{i}$ with a finite value, then this solution has a smooth continuation in the $\alpha<\alpha_{i}$ region. The $x_{0}$ solution increases in this case as $\alpha$ decreases and becomes infinite at the $\alpha$ root of the equation:

$$
\frac{|\delta|^{2}+\epsilon_{3}}{|\delta|}=\frac{\epsilon_{3} \cos (\vartheta+\theta)-\cos (\vartheta-\theta)}{\sin \vartheta} .
$$

\section{References}

Akhiezer, A. I., et al., High-frequency plasma oscillations, Proc. II U.N. Int. Conf. Peaceful Uses of Atomic Energy 31, 99-111 (United Nations, Geneva, 1958).

Allis, W. P., Motions of ions and electrons, Handbuch der Physik XXI, 383-444 (Springer-Verlag, Berlin, 1956).

Epstein, P. S., Theory of wave propagation in a gyromagnetic medium, Rev. Mod. Phys. 28, 3-17 (Jan. 1956).

MeLachlan, N. W., Bessel Functions for Engineers (Clarendon Press, Oxford, 1955).

Schumann, W. O., Über die Ausbreitung elektrischer Wellen längs einer dielektrisch begrenzten Plasmaschicht mit einem longitudinalem Magnetfeld, Z. Angew. Phys. 10, 26-31 (1958).

Smullin, L. D., and P. Chorney, Propagation in ion loaded waveguides, Proc. Symp. Electronic Waveguides, pp. 229-247 (Polytechnic Press, Brooklyn, 1958).

Trivelpiece, A. W., and R. W. Gould, Space charge waves in cylindrical plasma columns, J. Appl. Phys. 30, 1784-1793 (Nov. 1959).

(Paper 66D5-214) 